\title{
Searching for Extraterrestrial Technologies Within Our Solar System
}

\author{
Allen Tough \\ University of Toronto, 16 Balaby Crescent, Toronto, ON M3C-2B7 \\ Canada,E-Mail: AllenTough@aol.com \\ Guillermo A. Lemarchand \\ Instituto Argentino de Radioastronomía (CONICET) and Centro de \\ Estudios Avanzados (UBA), C.C. 8 - Sucursal 25, C1425FFJ Buenos \\ Aires, Argentina,E-mail: lemar@cea.uba.ar
}

\begin{abstract}
We speculate about possible cosmic exploration by extraterrestrial civilizations using interstellar smart probes. We call attention to some emerging technologies that may be used for interstellar exploration. We estimate that advanced civilizations may already have these technologies and may be using them to contact their galactic neighbors. We explore some observational consequences of these contacting strategies.
\end{abstract}

\section{Introduction}

Although we know almost nothing about the characteristics of extraterrestrial civilizations, we may assume that some of them might be very old and extraordinarily advanced. Because the age of the Sun is almost a third of the age of the universe, civilizations that have arisen around other stars are likely to be much older than ours. Our technology for interstellar communication is very young, largely developed in just the last century: any alien technology that we may detect using the terrestrial state-of-the-art technology will likely be thousands or even millions of years beyond our adolescent technology.

As we reflect on the likely capacities of civilizations much older than ours, it certainly seems possible that some of them have sent intelligent probes to other stars in order to monitor or dialogue with any civilizations that have arisen. Just as we send exploratory probes as far as we can in any given era, other civilizations will do the same. Some space scientists and engineers are exploring the theoretical possibility for the construction of interstellar probes that may travel at one-tenth the speed of light. Their feasibility is supported by today's cutting-edge work in robotics, artificial intelligence, nanotechnology and beam propulsion. If a few civilizations in our galaxy began sending intelligent probes to monitor various planetary systems thousands of years ago, then an intelligent alien probe could well have reached our solar system by now. We examine the array of past and potential strategies for detecting such a probe.

\section{When Technology Changes, New Search Paradigms Emerge}

Science and technology have changed greatly in the 40 years since the SETI field chose radio telescopes as its key strategy. Relying entirely on radio was a 
logical choice 40 years ago. Radio telescopes were just becoming popular among astronomers, two eminent scientists wrote a paper urging their use for SETI, and a distinguished engineer wrote a paper claiming that interstellar propulsion is impossibly slow and expensive. But our scientific and engineering knowledge today is dramatically different from what it was 40 years ago. Today's decisions about appropriate strategies should be based on the science and technology that we can confidently anticipate today, not on their status in 1959. Today's choices have to take into account our recent advances in such fields as computers, artificial intelligence, robotics, surveillance methods, molecular manufacturing (nanotechnology), propulsion, space exploration, lasers, and fiber optics.

In the past 30 years there have been several proposals of rapid space probes $(V \simeq 0.1 c$ ) for interstellar travel purposes, including pulsed fusion and antimatterpowered rockets, laser pushed light-sails and interstellar ramjets (Mallove \& Matloff 1989). Bracewell (1960) and Freitas (1980) have discussed the possible superiority of interstellar probes in missions of galactic exploration. Bracewell's 1960 paper proposed that an advanced extraterrestrial could send automatic space probes to neighbouring planetary systems to make contact with emerging technological societies. Papagiannis (1978), the founder of the IAU Bioastronomy Commission 51, proposed a search for alien artifacts in our solar system. Later he searched the IRAS data for objects in the asteroid belt with peculiar infrared spectra. Tipler (1980) has emphasized how easy it would be for an advanced civilization to explore the galaxy with self-replicating probes. Tough (1998) has written about the possibility of very small, very smart interstellar probes.

Most mainstream SETI research programs have ignored or dismissed the possibility of extraterrestrial space probes in our solar system based on the extrapolations of energy consumption of the fleet of space probes needed to explored a substantial region of the galaxy. A recent roadmap of technological developments applied to SETI up to 2020, for instance, devotes just a few sentences to the possibility of trying to detect interstellar probes (Ekers et al. 2002). Out-of-date extrapolations are based on the assumption of $20^{\text {th }}$ Century payloads and fail to consider how cheap interstellar probes will be once a civilization has mastered nanotechnology, artificial intelligence, and interstellar propulsion. Such developments also indicate that the Chiu-Sagan paradox (Chiu 1970; Sagan 1972) may be wrong. If at least one civilization in our galaxy developed advanced technologies long ago, it is quite possible that at least one smart interstellar probe has reached our solar system.

\section{Future Search Possibilities Within the Solar System}

Over the next few years, how might scientists search within the solar system for unassailable evidence of an alien object. This object might be a probe or spacecraft, for instance, or its discarded parts. Such a search might focus on the Moon, the asteroid belt, or the Lagrange equilibrium points. Alternatively, the alien object might be a building, a monument, or some other artificial structure. Indeed, an alien intelligence may have deliberately left an artifact for us to discover at some special landmark in the solar system, such as the highest point on Mars or the deepest canyon on Venus, or in some carefully chosen spot that we will explore someday because of our scientific curiosity or our appreciation of 
beauty. Or equipment might have been stored below the surface of some body (perhaps in natural cracks or passages) to protect it from damage by cosmic radiation and micrometeorites.

Another approach is to search for heat, exhaust gases or other effluents and byproducts that might be emitted by a probe or spacecraft. Looking for IR anomalies in the asteroid belt is often suggested as one example, or trying to detect communications from the probe back to its home base, or analyzing with the KLT mathematical transform some peculiar EM signals (Maccone 1994) that may indicate the signature of a rapidly decelerating alien spacecraft.

Another possibility is to search for evidence of mining (mainly in the asteroid belt), in case probes have mined the materials necessary to construct self-replicating probes or the so-called von Neumann machines. This idea has been widely discussed in the SETI literature over the years.

Another option is to search the planet Earth for physical evidence of an alien object. It might be in orbit, on land, or in an ocean. It might have arrived recently or millions of years ago. It might be a super-intelligent probe that is actively monitoring us, or simply a discarded part from an ancient probe. One possibility is that a very small smart probe is hidden among the space debris that is orbiting around the Earth: it might be extremely difficult to identify. Various sciences, and of course various intelligence and security agencies, are already monitoring much of the Earth, including the oceans and space. Or a hiker or recreational diver may discover an alien object by serendipity, just as an astronomer may someday detect an astroengineering object or any other artificial signal while studying some other natural phenomenon.

\section{A Fresh Alternative to Physical Detection}

In the past few years, SETI scientists have realized how incredibly smart, knowledgeable, autonomous, and competent an interstellar probe might be. Imagine the technological capacity that we humans (and our smart robots) will develop in the next 100 years. Then extrapolate for many more centuries to get some idea of the potential powers of alien technology. In contrast, some earlier proposals seemed to assume an alien probe is not very smart. Sometimes, for instance, it was viewed as a tape-recorder that will play a message over and over if triggered by a predetermined impulse.

A few far-sighted scientists and engineers, particularly Kurzweil (1999) and Moravec (1999), have shaken up our thinking. They spell out, decade by decade, just how we might develop smarter-than-human robots before the end of this century. If an alien civilization is many centuries older than us, it might have developed super-smart robots long ago. Especially if these are extremely small and lightweight thanks to molecular manufacturing (nanotechnology), they might be sent off to explore other planetary systems. At least one of these small, smart, autonomous interstellar probes may have already reached our solar system.

As we adjust to the idea of an incredibly smart knowledgeable probe-as we allow ourselves to deeply grasp the implications of an intelligence that is millions of years beyond ours-a fresh strategy comes to mind. Instead of trying to physically detect this probe, perhaps by sneaking up on it or outwitting it, we can invite it to interact with us. 
Presumably a technology thousands or millions of years beyond ours can cloak itself successfully. And presumably it will elude our primitive efforts to outwit it. Rather than being stymied by the probe's highly advanced capacities, however, a fresh approach can build on them. It can assume a super-smart probe will readily monitor our telecommunications, learn our major languages, and figure out how to navigate our World Wide Web and its search engines. The fresh approach simply places an invitation to ETI on the Web-an invitation to dialogue with all of humankind. If a super-smart probe looks up "extraterrestrial intelligence" or "alien probe" in the major search engines, it will discover the invitation. In October 1998, this approach was implemented by a group of SETI scientists as the "Invitation to ETI" (http://members.aol.com/WelcomeETI).

\section{Concluding Summary}

- As civilizations and their technology advance, they eventually develop the capacity to manufacture smart autonomous probes to explore the galaxy.

- Such a probe may already have reached our solar system. The SETI field should strongly support any scientific efforts to find irrefutable evidence of such a probe.

- Because a probe may be extraordinarily intelligent-perfectly capable of monitoring our WWW-we should issue an invitation to a dialogue.

- The Invitation to ETI has a reasonable chance of detecting ETI, at least as good as any other SETI project.

Acknowledgments. GAL thanks Allen Tough and IAU for the financial support to attend this Symposium.

\section{References}

Bracewell, R. N. 1960, Nature, 186, 670

Chiu, H. Y. 1970, Icarus, 11, 447

Ekers, R. D., Cullers, D. K., Billingham, J., \& Scheiffer, L. K. ed. 2002, SETI 2020: A Roadmap for the Search for Extraterrestrial Intelligence, (Mountain View: SETI Press), pages 2-3, 113, 230

Freitas, R. A. 1980, J. of the British Interplanetary Society, 33, 95

Kurzweil, R. 1999, The Age of Spiritual Machines: When Computers Exceed Human Intelligence, (New York: Viking)

Maccone, C. 1994, Telecommuncations, KLT and Relativity, (Colorado Springs: IPI Press)

Mallove, E. F., \& Matloff, G. L. 1989, The Starflight Handbook: A Pioneer's Guide to Interstellar Travel, (New York: Wiley)

Moravec, H. 1999, Robot: Mere Machine to Transcendent Mind, (New York: Oxford University Press)

Papagiannis, M. D. 1978, Quart. J. of R. Astron. Soc., 19, 217

Sagan, C. 1972, UFO's the Extraterrestrial and Other Hypothesis in UFO's: A Scientific Debate, Sagan and Page eds., (Cornell University Press: Ithaca)

Tipler, F. J. 1980, Quart. J. of R. Astron. Soc., 21, 267

Tough, A. 1998, J. of the British Interplanetary Society, 51, 167 